\title{
Formation of Artificial Granules for Proving Gelation as the Main Mechanism of Aerobic Granulation in Biological Wastewater Treatment
}

\author{
Yun Li and Xiao-yan $\mathrm{Li}^{*}$ \\ Environmental Engineering Research Centre, Department of Civil Engineering, The University of \\ Hong Kong, Pokfulam, Hong Kong, China \\ (E-mails: u3001701@hku.hk; xlia@hkucc.hku.hk)
}

\begin{abstract}
In this study, gelation-facilitated biofilm formation as a new mechanism is proposed for the phenomenon of aerobic granulation in biological wastewater treatment. To obtain an experimental proof for the gelation-based theory, the granulation process was simulated in a chemical system using latex particles for bacterial cells and organic polymers (alginate and peptone) for extracellular polymeric substances (EPS) in a solution with the addition of cations $\left(\mathrm{Ca}^{2+}, \mathrm{Mg}^{2+}\right.$ and $\left.\mathrm{Fe}^{3+}\right)$. The results showed that at a low alginate content $\left(70 \mathrm{mg} \cdot \mathrm{g}^{-1} \mathrm{MLSS}\right)$ flocculation was observed in the suspension with loose flocs. At a higher alginate content (180 $\mathrm{mg} \cdot \mathrm{g}^{-1}$ MLSS), together with discharge of small flocs, formation of artificial gel granules was successfully achieved leading to granulation. The artificial granules show a similar morphological property as actual microbial granules. However, if the protein content increased, granulation became difficult with little gel formation. The experimental work demonstrates the importance of the bonding interactions between EPS functional groups and cations in gel formation and granulation. The laboratory results on the formation of artificial granules provide a sound proof for the theory of gelation-facilitated biofilm formation as the main mechanism for aerobic granulation in sludge suspensions.
\end{abstract}

Keywords

Activated sludge; aerobic granules; biological wastewater treatment; extracellular polymeric substances (EPS); gelation; granulation

\section{INTRODUCTION}

Aerobic sludge granulation is a new biological wastewater treatment technology. The aerobic granular process has attracted increasing research attentions in recent years owing to its advantages over the conventional activated sludge process, such as high biomass density, fast settling velocity, rapid sludge-effluent separation and strong resistance to shock loading and toxic chemicals (Adav et al., 2008; Ho et al., 2010; Xiao et al., 2008). Various operational parameters have been proposed as important means for aerobic granulation, including a short settling time, strong hydraulic shear intensity and the feast-famine regime (Liu et al., 2004; Adav et al., 2008). The impetus of excess extracellular polymeric substances (EPS) together with rapid sludge settling and sufficient sludge discharge appear to be crucial to granule formation and growth. EPS are viscid polymers that can adhere to cells to form biofilms with a dense structure. EPS mainly consist of polysaccharides, proteins, humic acids, lipids and nucleic acids, and the first two types of macromolecules, polysaccharides and proteins, are believed to play a significant role in aerobic granulation (Durmaz and Sanin, 2001). Effort had been made to relate the abundances of polysaccharides and proteins as well as their ratio (PS/PN) to the granulation process in bioreactors (Tay et al., 2001; McSwain et al., 2005). 
Use of macromolecular polymers, such as polysaccharides and proteins, in food industry can result in hydrogel formation (Totosaus et al., 2002). Aerobic granules may be considered as hydrogels as they share many similar properties, e.g. a compact structure, a regular shape, smooth surface and a water swollen body. Seviour et al. (2009) have presented a strong evidence by showing the storage and loss moduli that aerobic granules are physically hydrogels while flocs do not own gel properties. Gelation is a process of gel particle formation, or material solidification, in a solution at ambient temperature. Lin et al. (2010; 2013) extracted alginate-like exopolysaccharides (ALE) from aerobic granules and demonstrated the gel-forming property of ALE in a cation (e.g. $\mathrm{CaCl}_{2}$ ) solution. The mechanism of ALE-based gelation has been explained by the bonding between carboxylic acids and divalent metal ions based on the classical egg-box model that was first proposed by Morris et al. (1982).

However, most previous research has focused on the gel properties of aerobic granule with little attention on the dynamic process of gel formation. In particular, the transformation from loose flocs to dense granules has not been exactly demonstrated in a well defined chemical system. In this study, in relation to aerobic granulation, a lab experiment was carried out to simulate the gel formation process in a particle suspension with the alginate and peptone additions. The study was designed to form artificial granules via gelation to provide an experimental proof to the theory of gelation-facilitated biofilm formation for the mechanism of aerobic sludge granulation.

\section{MATERIALS AND METHODS}

\section{Cultivation of aerobic granules}

Batch column reactors were used to cultivate aerobic granules at room temperature $\left(20-22^{\circ} \mathrm{C}\right)$. Each column had a dimension of $3.5 \mathrm{~cm}$ in diameter and $30 \mathrm{~cm}$ in height with a working volume of $200 \mathrm{ml}$ (Figure 1). The synthetic wastewater consisting of glucose as the carbon source and nutrients was prepared according to the formula given by Tay et al. (2001). Seed sludge was an activated sludge suspension collected from a full-scale domestic wastewater treatment plant (Stanley Sewage Treatment Works, Hong Kong). The sludge mixture had a MLSS concentration of $3500 \mathrm{mg} / \mathrm{L}$ with a VSS/SS ratio of 0.68 . The reactor was fed manually twice a day with synthetic wastewater after the effluent discharge. The influent chemical oxygen demand (COD) loading increased gradually from 700 to $4000 \mathrm{mg} \cdot \mathrm{L}^{-1} \cdot \mathrm{d}^{-1}$, meanwhile the settling time was shortened from 30 to 1 min to keep fast-settling flocs for development of granules. Fine bubbles were pumped into the reactor through an air diffuser at about $0.8 \mathrm{~L} \cdot \mathrm{min}^{-1}$ during the aeration phase. The $\mathrm{pH}$ in the reactor was maintained in the neutral range between 7.0 and 7.5 by adding $\mathrm{NaHCO}_{3}$.

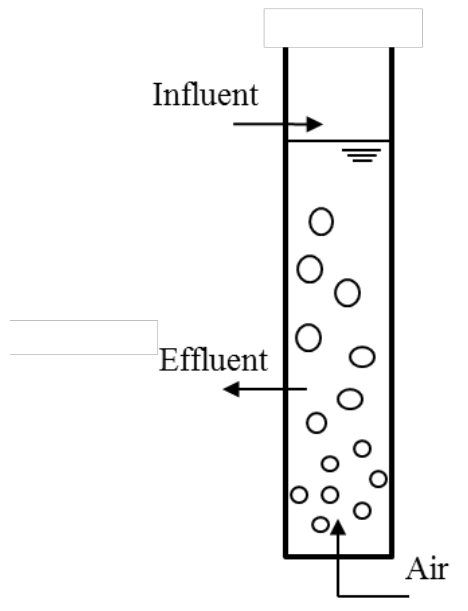

(a)

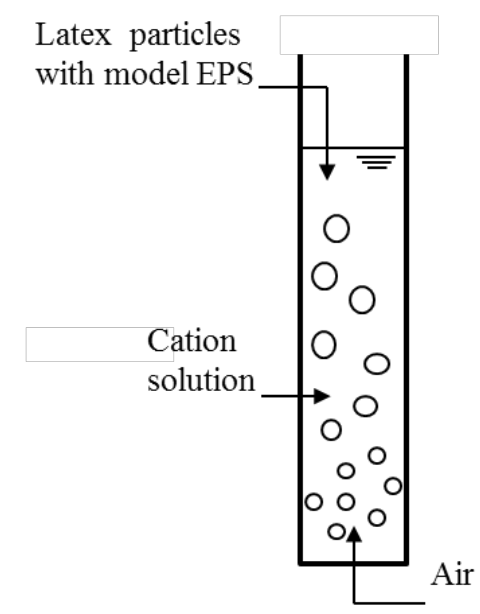

(b)

Figure 1. Columns used for (a) a biological reactor to cultivate aerobic granules and (b) a chemical reactor to form artificial granules. 


\section{Formation of artificial granules for simulation of microbial granulation}

The columns described above were also employed for the experimental tests on gel formation and granulation simulation. Latex microspheres with an average diameter of $0.99 \mu \mathrm{m}$ and a density of $1.05 \mathrm{~g} \cdot \mathrm{cm}^{-3}$ were suspended in water to simulate bacterial cells. Alginate and peptone were used as representative polysaccharides and proteins in microbial EPS, respectively. These model EPS components were dosed in different combinations and dosages, including alginate alone, peptone alone, and a mixture of alginate and peptone. The cation solution consisted of $\mathrm{CaCl}_{2}, \mathrm{MgCl}_{2}$ and $\mathrm{FeCl}_{3}$ at a concentration of $3.7 \mathrm{mmol} \cdot \mathrm{L}^{-1}$ with a $\mathrm{Ca}^{2+}: \mathrm{Mg}^{2+}: \mathrm{Fe}^{3+}$ ratio of $5: 4: 1$, which is similar to that in municipal wastewater. Latex particles were first socked in the model EPS solutions to allow the adsorption of polymers on the particle surface, which simulated bacteria with secreted EPS surrounded. The treated particles were then placed into the cation solution with aeration provided to promote particle aggregation and clustering. After a short period of settling, slow settling particle flocs were discharged from the column. In the remaining solution in the column, more latex particles with model EPS polymers were added. Afterward, the particle suspension was added into the cation solution to induce particle aggregation and attachment. The procedures were repeated till the gel formation was observed and artificial granules were formed.

\section{Characterization of aerobic granules and artificial granules}

The microbial granules and artificial granules were examined using a scanning electron microscope (SEM). Both granule samples were first fixed by formaldehyde (4\%) before dehydrated by ethanol through a concentration gradient. Before observation with the SEM (Hitachi S3400N VP), critical point drying and lyophilization was used to dry both artificial granules and aerobic granules (Gerhardt et al., 1994).

Artificial flocs and granules were further analyzed by a Fourier transform infrared (FTIR) spectrometer. The lyophilized flocs or granules were mixed with $\mathrm{KBr}$ power at a ratio of 1:1 and homogenized in an agate grinder. One hundred and fifty mg of the solid mixture was compressed and analyzed with a spectrum 8900 IR spectrometer (Shimadzu, Japan). The scanning condition was the same as that described by Hong et al. (1999).

\section{Analysis methods}

Measurements of COD and mixed liquid suspended solids (MLSS) were performed in accordance with Standard Methods (1998). For the artificial flocs and granules, the determination of solid contents was the same as the MLSS measurement. The apparent density of granules was determined with a series of glucose solutions of different concentrations and densities (Su and Yu, 2005).

\section{RESULTS AND DISCUSSION}

\section{Cultivation of aerobic granules}

Aerobic granules were produced from the seed activated sludge flocs after 40 days of cultivation. During the granulation process, the organic loading and MLSS concentrations increased with time (Figure 2). The size of mature granules was between 2-3 mm. Compared to activated sludge flocs, aerobic granules are larger and denser with a smooth surface (Figure 3). 


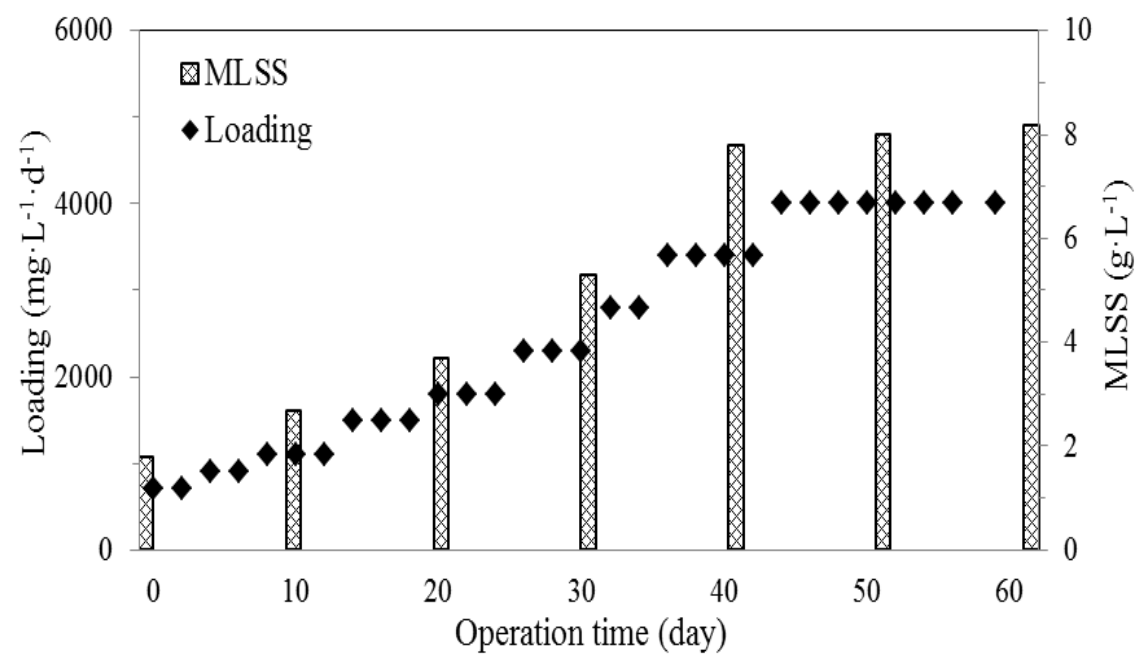

Figure 2. Organic loading and sludge concentration during the cultivation of aerobic granules.
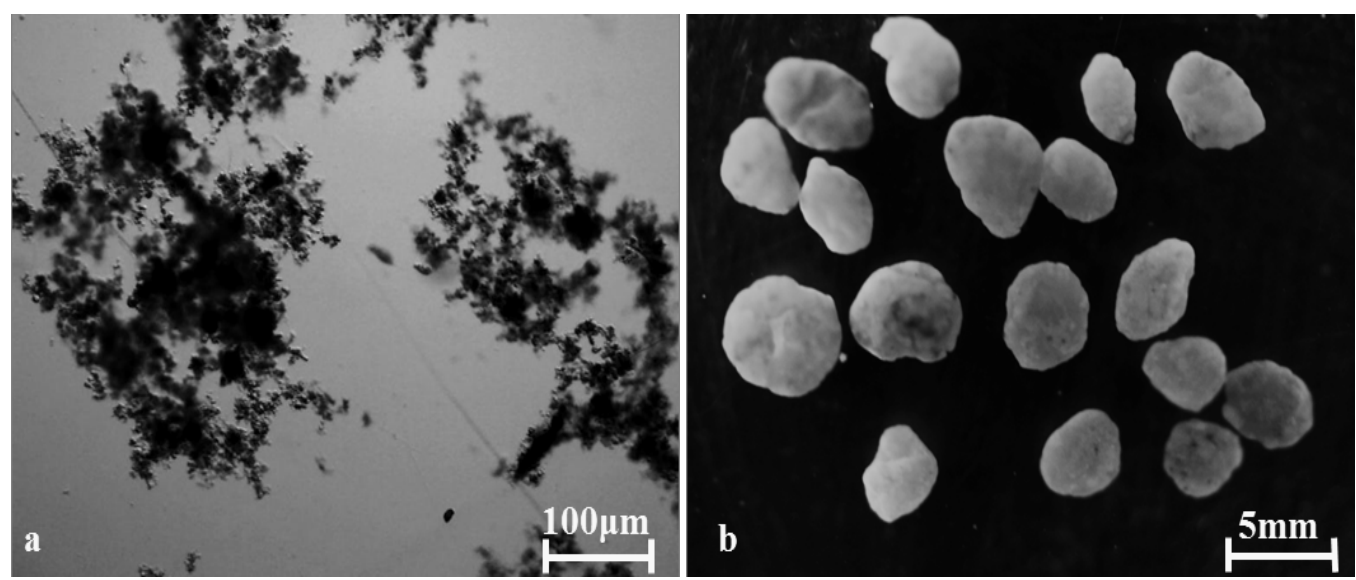

Figure 3. Images of (a) seed activated sludge flocs and (b) mature aerobic granules.

\section{Formation of artificial granules via gelation}

Artificial EPS consisting of peptone alone. Latex microspheres are used to simulate bacterial cells, and peptone was used as artificial EPS that was adsorbed by the latex particles. When the particle suspension was placed into the cation solution, aggregation of the particles occurred in the mixture. The peptone content increased from 70 to $500 \mathrm{mg} \cdot \mathrm{g}^{-1}$ MLSS during the study. For each experimental test, the particles and cation solutions were added repeatedly into the suspension, whilst the supernatant with suspended particles and small flocs were discharged. The results show that only the formation of particles flocs was observed for the samples taken at different stages after various feeding and discharging cycles (Figure 4). The particle flocs were similar in appearance to activated sludge flocs. The size of the flocs increased during the dosing and flocculation process; however, the flocs were loose in structure without any sign of gel formation. It has been reported that the concentrations of proteins in EPS extracted from aerobic granulation process are lower than 200 $\mathrm{mg} \cdot \mathrm{g}^{-1}$ MLSS (McSwain et al., 2005). In Figure 4d, the peptone concentration was increased to 500 $\mathrm{mg} \cdot \mathrm{g}^{-1}$, far beyond reach of bacteria secretion; however, little gel formation was observed. It is apparent that peptone has little ability to form gel with cations, for which granulation is difficult to achieve. 

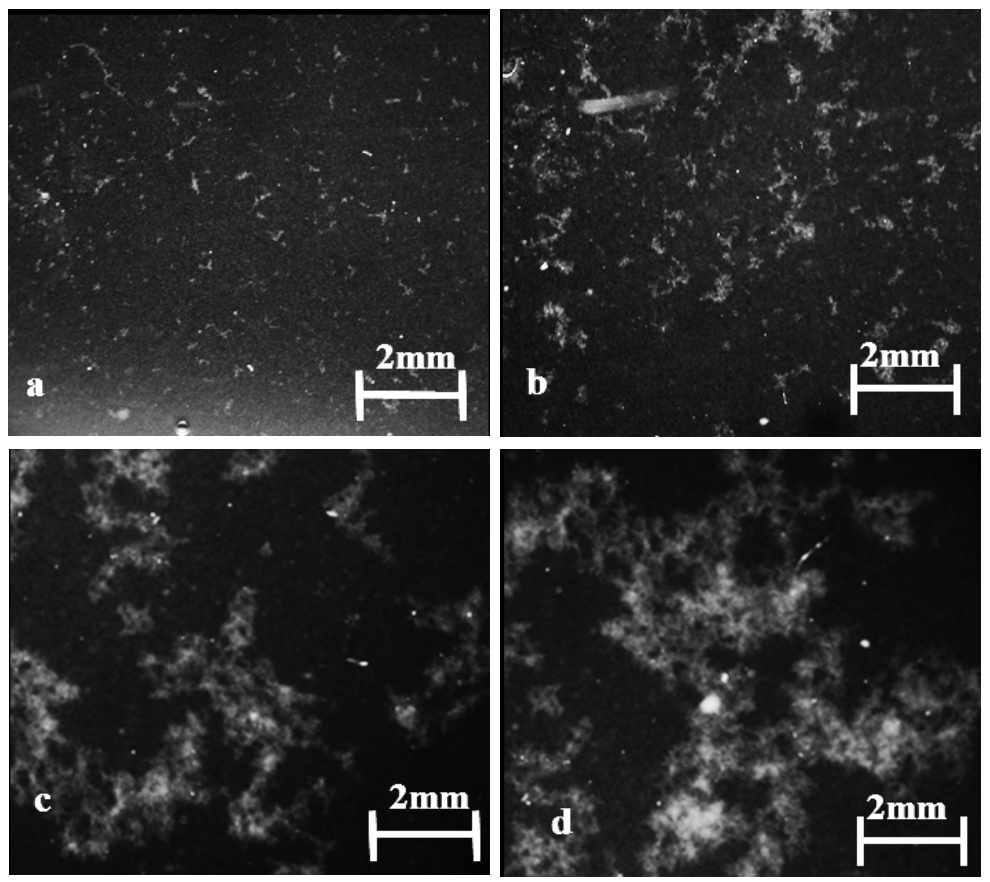

Figure 4. Images of the flocculation products after (a) 12 cycles, (b) 15 cycles, (c) 22 cycles and (d) 25 cycles.

In food industry, heating or high pressure is often needed for proteins to form gels (Totosaus et al., 2002), which is not feasible for the lab reactors used in the present study or sludge suspensions for biological wastewater treatment. The results imply that proteins can play an important role in microbial flocculation. However, proteins alone are not sufficient to lead to bacterial gelation and gelation-based granulation.

Artificial EPS consisting of alginate alone. Alginate was used to represent polysaccharides in EPS secreted by cells. The alginate was adsorbed by the latex microspheres, and the alginate contents from 70 to $180 \mathrm{mg} \cdot \mathrm{g}^{-1}$ MLSS were tested, which is comparable to the actual EPS content in granules (Durmaz and Sanin, 2001). With the presence of cations, granule formation was successfully simulated in this particle system (Figure 5). Small flocs were observed after 7 cycles. By increasing the alginate content, together with the discharge of single particles and small flocs, the flocs retained in the suspension become bigger and denser after 10 cycles (Figure 5b and 5c). Eventually, gelation was achieved and artificial granules with a clear surface were formed (Figure $5 \mathrm{~d}$ ). The granules were round gels sizing between 3-4 mm with a density of $1.06 \sim 1.08 \mathrm{~g} \cdot \mathrm{cm}^{-3}$, which are similar to aerobic granules. The experimental results with alginate demonstrated that transformation from flocs to granules could be achieved by gelation.

It is generally accepted that extracellular polysaccharides play a key role in cohesion of cells and granulation (Adav et al., 2008). Exopolysaccharides could form a three-dimensional structure by adhesion of bacteria, so that more cells and particles would be attached together into a bigger and denser community (Tay et al., 2001). In this simulation, gelation is proven to be an effective way for polysaccharides to form gels and to attach other particles and small flocs, evolving into granules. 

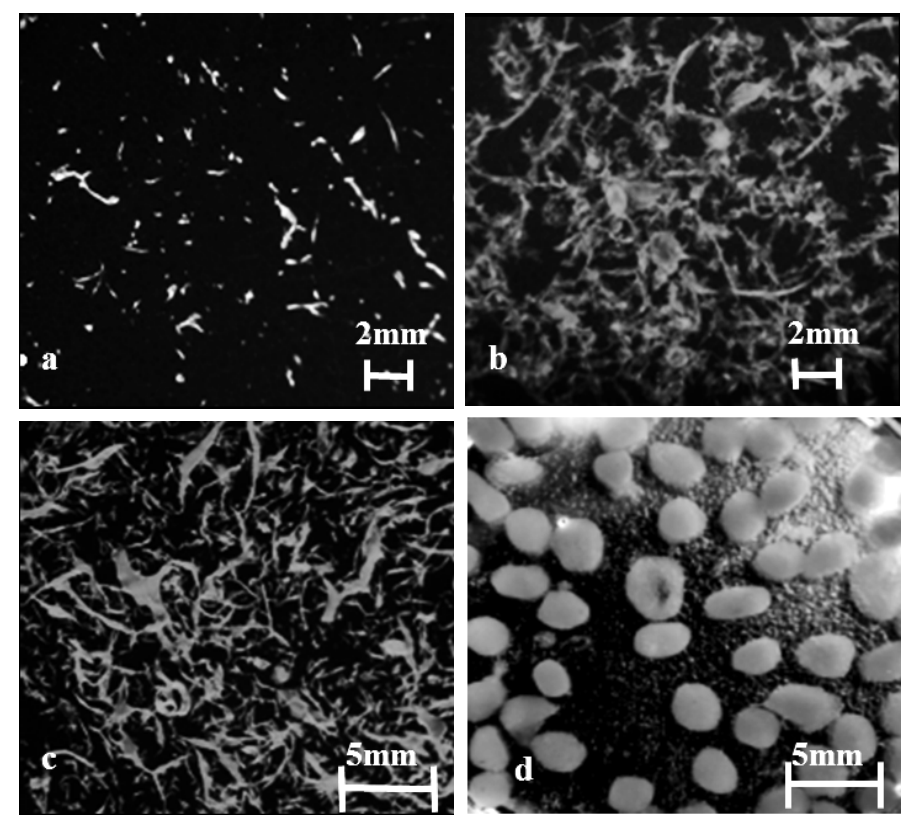

Figure 5. Images of the gel products after (a) 2 cycles, (b) 7 cycles, (c) 10 cycles and (d) 14 cycles.

Artificial EPS consisting of both alginate and peptone with different PS/PN ratios. Alginate and peptone were mixed at a series of PS/PN concentration ratios from 2.6 to 5.7, and the total EPS content increased from 48 to $135 \mathrm{mg} \cdot \mathrm{g}^{-1}$ MLSS. Artificial granules with a smooth surface and clear shape were produced in the reactors after 16 feeding and discharging cycles. Thus, a high PS/PN ratio is favorable to granules formation and growth, as polysaccharides make more contribution to granulation. However, in the presence of both alginate and peptone, compared to the experiment with only alginate, both the amount of the EPS contents and the time for granule formation became less in comparison to the experiment with alginate alone. It is apparent that, while alginate helped form the backbone of gels, the hydrophobicity of proteins would accelerate gel formation and granulation.

In contrast, when a low PS/PN ratio from 0.5 to 0.28 was applied, even though the total EPS content increased to $300 \mathrm{mg} \cdot \mathrm{g}^{-1} \mathrm{MLSS}$, artificial granules could not be formed in the system. The results suggest that granulation could be hardly achieved with the EPS dominated by proteins.

\section{Characterization of the artificial granules}

SEM examination of aerobic granules and artificial granules. According to SEM observations, aerobic granules have a tightly packed structure, and the bacteria in granules were surrounded by apparent EPS matrix (Figure 6). Similarly, the artificial granules have a denser structure, and the latex particles were immobilized with threadlike artificial EPS. The similar morphology between actual aerobic granules and artificial gel granules evidenced a successful simulation of the granulation process in a simple particle system under a well-controlled laboratory condition.
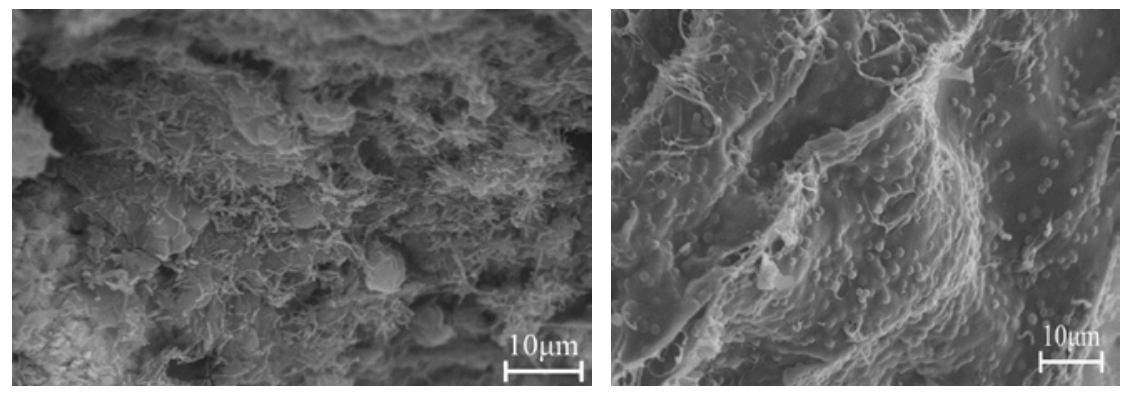

Figure 6. SEM images of aerobic granules (left) and artificial gel granules (right). 
FTIR analysis of the artificial granules. The flocs and granules formed by the latex particles with artificial EPS were analyzed with the FTIR (Figure 7). The $\mathrm{COO}^{-}$peaks could be identified at around $1640 \mathrm{~cm}^{-1}$ and $1380 \mathrm{~cm}^{-1}$ (asymmetric and symmetric stretch, respectively), which became broader for the artificial granules than flocs. In addition, compared to the flocs, the $\mathrm{COO}^{-}$peaks of the granules shifted to certain degrees towards higher wavelengths. The shift suggests the formation of new coordinate bonding between the carboxylic group and metal ions. That is, according to the egg-box model, cations can take the places of sodium ions in alginate to form a new bonding. The substitution would create a new electron distribution, resulting in the peak shifts. Meanwhile, the C-O stretching peak around $1088 \mathrm{~cm}^{-1}$ shifted to lower wavelengths. This is likely caused by the formation of new metal-carboxyl coordinate bonding that weakened the C-O bonds (Sartori et al., 1997).

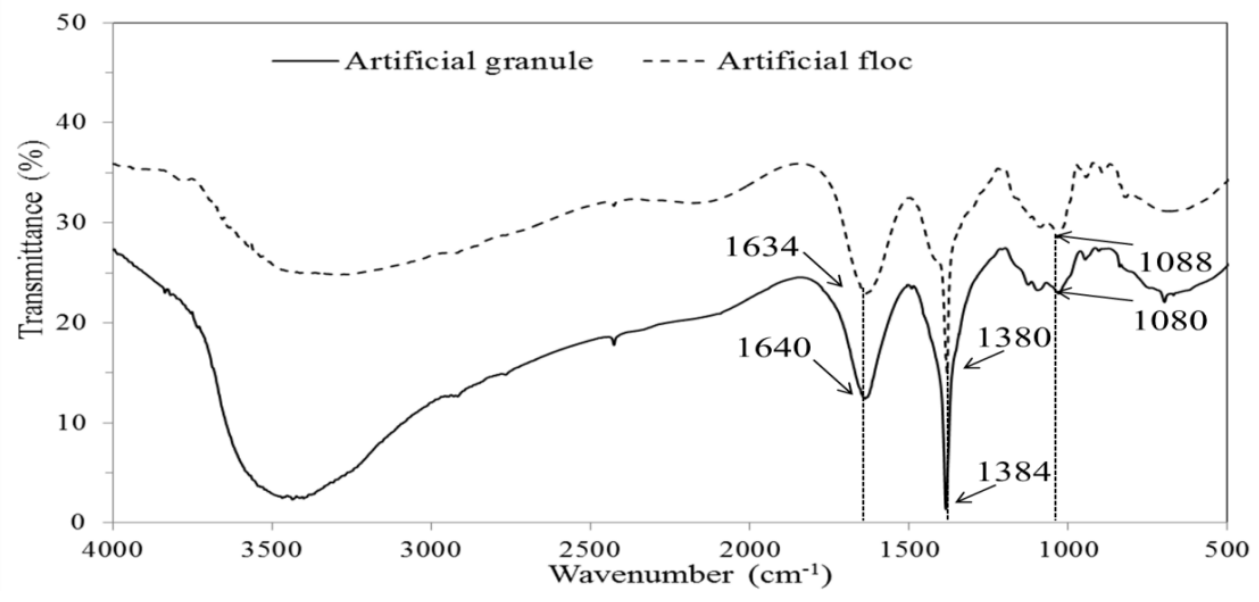

Figure 7. FTIR spectra of the artificial flocs and granules.

The experimental work in a well-controlled chemical system demonstrated the formation of granules via gelation in a particle suspension. The simulation results imply that gel-facilitated biofilm formation is a sound explanation to aerobic granulation in bioreactors. EPS polymers can form gels through coordinate bonding with metal ions in wastewater, leading to granule formation. Thus, the research findings provide a solid experimental proof for the gelation-based mechanism of aerobic granulation in biological wastewater treatment.

\section{CONCLUSIONS}

In a simple particle system with latex microspheres for bacterial cells and alginate and peptone for EPS, the granulation process can be simulated with the cation addition and discharge of particles and small flocs. The artificial granules showed a similar morphological property as actual microbial granules. Alginate is crucial to gel formation via the coordinate bonding, while peptone can accelerate the process. The results prove the essential role of EPS polysaccharides and their interactions with cations in gel formation, which leads to aerobic granulation. The experimental work on the formation of artificial granules provides a sound proof for the theory of gelation-based biofilm formation as the main mechanism for aerobic granulation in sludge suspensions.

\section{ACKNOWLEDGEMENTS}

This research was supported by grant 51129803 from the Natural Science Foundation of China, grants HKU714811E from the Research Grants Council (RGC) and SEG_HKU10 from the University Grants Committee (UGC) of the Hong Kong SAR Government. The technical assistance of Mr. Keith C.H. Wong is highly appreciated. 


\section{REFERENCES}

Adav S.S., Lee D.-J., Show K.-Y. and Tay J.-H. (2008). Aerobic granular sludge: Recent advances. Biotechnol. Adv. 26(5), 411-423.

Durmaz B. and Sanin F.D. (2001). Effect of carbon to nitrogen ratio on the composition of microbial extracellular polymers in activated sludge. Water Sci. Technol. 44(10), 221-229.

Gerhardt P., Murray R.G.E., Wood W.A. and Krieg N.R. (1994). Methods for General and Molecular Bacteriology, Washington D.C., U.S.A.

Ho K.-L., Chen Y.-Y., Lin B. and Lee D.-J. (2010). Degrading high-strength phenol using aerobic granular sludge. Appl. Microbiol. Biotechnol. 85(6), 2009-2015.

Hong K., Sun S., Tian W., Chen G. and Huang W. (1999). A rapid method for detecting bacterial polyhydroxyalkanoates in intact cells by Fourier transform infrared spectroscopy. Appl. Microbiol. Biotechnol. 51(4), 523-526.

Lin Y., de Kreuk M., van Loosdrecht M.C.M. and Adin A. (2010). Characterization of alginate-like exopolysaccharides isolated from aerobic granular sludge in pilot-plant. Water Res. 44(11), 3355-3364.

Lin, Y. M., Sharma, P. K. and van Loosdrecht, M. C. M. (2013). The chemical and mechanical differences between alginate-like exopolysaccharides isolated from aerobic flocculent sludge and aerobic granular sludge. Water Res. 47 (1), 57-65.

Liu Y.-Q., Liu Y. and Tay J.-H. (2004). The effects of extracellular polymeric substances on the formation and stability of biogranules. Appl. Microbiol. Biot. 65(2), 143-148.

McSwain B., Irvine R., Hausner M. and Wilderer P. (2005). Composition and distribution of extracellular polymeric substances in aerobic flocs and granular sludge. Appl. Environ. Microb. 71(2), 1051-1057.

Morris E., Powell D., Gidley M. and Rees D. (1982). Conformations and interactions of pectins: I. Polymorphism between gel and solid states of calcium polygalacturonate. J. Mol. Biol. 155(4), 507-516.

Sartori C., Finch D.S., Ralph B. and Gilding K. (1997). Determination of the cation content of alginate thin films by FTi.r. spectroscopy. Polymer 38(1), 43-51.

Seviour T., Pijuan M., Nicholson T., Keller J. and Yuan Z. (2009). Understanding the properties of aerobic sludge granules as hydrogels. Biotechnol. Bioeng. 102(5), 1483-1493.

Standard Methods for the Examination of Water and Wastewater. 1998. 20nd Edition. Rice, E.W., Baird, R.B., Eaton, A.D., Clesceri, L.S., eds. American Public Health Association, Washington, DC.

Su K.Z. and Yu H.Q. (2005). Formation and characterization of aerobic granules in a sequencing batch reactor treating soybean-processing wastewater. Environ. Sci. Technol. 39(8), 2818-2827.

Tay J.H., Liu Q.S. and Liu Y. (2001). The role of cellular polysaccharides in the formation and stability of aerobic granules. Lett. Appl. Microbiol. 33(3), 222-226.

Totosaus A., Montejano J.G., Salazar J.A. and Guerrero I. (2002). A review of physical and chemical protein-gel induction. Internat. J. Food Sci.Technol. 37(6), 589-601.

Xiao F., Yang S.F. and Li X.Y. (2008) Physical and hydrodynamic properties of aerobic granules produced in sequencing batch reactors. Separation Purification Technol., 63(3), 634-641. 